\title{
Evaporative gold nanorod assembly on chemically stripe-patterned gradient surfaces
}

\author{
Imtiaz Ahmad ${ }^{a}$, H. Patrick Jansen ${ }^{a}$, Jeroen van Swigchem ${ }^{a}$, Christian Ganser ${ }^{\text {b,c }}$, Christian Teichert ${ }^{\mathrm{b}}$, \\ Harold J.W. Zandvliet ${ }^{\mathrm{a}}$, E. Stefan Kooij ${ }^{\mathrm{a}, *}$ \\ a Physics of Interfaces and Nanomaterials, MESA+ Institute for Nanotechnology, University of Twente, PO Box 217, 7500AE Enschede, The Netherlands \\ ${ }^{\mathrm{b}}$ Institute of Physics, Montanuniversitaet Leoben, Franz-Josef-Strasse 18, A-8700 Leoben, Austria \\ ' Christian Doppler Laboratory for Surface Chemical and Physical Fundamentals of Paper Strength, Graz University of Technology, 8010 Graz, Austria
}

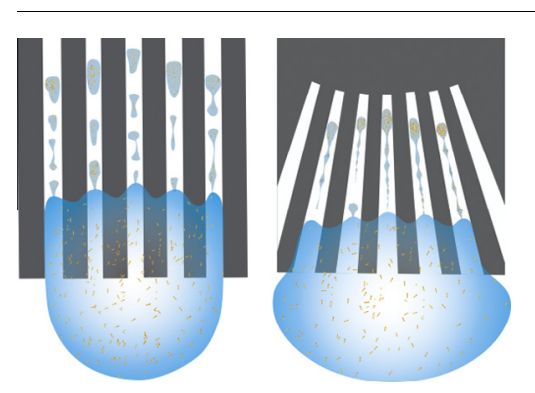

\section{A R T I C L E I N F O}

\section{Article history:}

Received 1 November 2014

Accepted 19 December 2014

Available online 30 December 2014

\section{Keywords:}

Gold nanorods

Directional wetting

Evaporative assembly

\begin{abstract}
A B S T R A C T
Experimentally we explore the potential of using pre-defined motion of a receding contact line to control the deposition of nanoparticles from suspension. Stripe-patterned wettability gradients are employed, which consist of alternating hydrophilic and hydrophobic stripes with increasing macroscopic surface energy. Nanoparticle suspensions containing nanorods and nanospheres are deposited onto these substrates and left to dry. After moving over the pattern and evaporation of the solvent, characteristic nanoparticle deposits are found. The liquid dynamics has a pronounced effect on the spatial distribution. Nanoparticles do not deposit on the hydrophobic regions; there is high preference to deposit on the wetting stripes. Moreover, the fact that distributed nanoparticle islands are formed suggests that the receding of the contact line occurs in a stick-slip like fashion. Furthermore, the formation of liquid bridges covering multiple stripes during motion of the droplet over the patterns is modeled. We discuss their origin and show that the residue after drying, containing both nanoparticles and the stabilizing surfactant, also resembles such dynamics. Finally, zooming into individual islands reveals that highly selective phase separation occurs based on size and shape of the nanoparticles.
\end{abstract}

(c) 2014 Elsevier Inc. All rights reserved.

\footnotetext{
* Corresponding author. Fax: +31534891101. E-mail address: e.s.kooij@utwente.nl (E.S. Kooij). URL: http://www.utwente.nl/tnw/pin (E.S. Kooij).
}

\section{Introduction}

\subsection{Nanoparticle assembly}

A highly interdisciplinary field of research within materials science comprises that devoted to nanomaterials. Macroscopic materials built from nanoscale building blocks derive their 
properties from those of the individual composing elements [1-7]. The bottom-up approach to design and manufacture novel nanoscale materials has rapidly matured over the past years. Impressive achievements in terms of well-accessible, reproducible synthesis procedures have led to the availability of a large diversity of monodisperse colloidal metallic, semiconducting and insulating particles with sizes ranging from micrometers to nanometers. Active and reproducible control over orientation and mutual alignment of nanoparticles in suspension as well as during adsorption at surfaces has mainly been limited to (controlled) drying experiments, in which hydrodynamic attractive forces compete with steric and/or electrostatic repulsive forces [7-16]. Additionally, the application of external stimuli, such as electric, magnetic and optical fields have been explored [17-24].

One of the most frequently used methods to deposit nanoparticles on substrates, referred to as evaporative self-assembly [25-27], involves drying of a droplet containing nanoparticles on an isotropic homogeneously coated substrate. This generally gives rise to concentrated deposition within a dense ring near the threephase contact line, the so-called coffee stain effect [9,28-30]. In a recent study [31] on evaporative assembly of mixed suspensions containing nanorods and nanospheres, we focused on the phaseseparation and alignment of the nanoparticles within this coffee strain ring. Depending on the position relative to the pinned contact line of the drying droplet, spheres and rods separate into various liquid-crystalline phases [31]. Experimental observations were compared to quantitative calculations of the colloidal interaction energies, and the role of the surfactant on the different crystal facets of the nanorods was discussed.

\subsection{Wettability gradients}

Despite the vast amount of work in the field of nanoparticle self-assembly, reproducible control over the nanoparticles in suspension as well as during their assembly at interfaces still poses major challenges. The aforementioned pinning of the contact line during the evaporation, i.e. the coffee stain effect, hinders the homogeneous deposition of nanoentities over large surface areas. Consequently, exploring alternative approaches to isotropic nanoparticle deposition is a timely topic of research. As an example, recently it has been shown that the coffee stain effect can be suppressed using electrowetting [32,33].

In that respect, assembly of nanoparticles by a moving contact line is particularly interesting since it enables deposition over much larger areas. A way to achieve controlled motion of the contact line is by using substrates with a coating that defines a gradient in the wetting properties [34-37]. In recent work, we have shown that on morphologically flat but chemically patterned surfaces such wettability gradients can be created using striped patterns of alternating hydrophilic and hydrophobic stripes [38-40]. Linear and radial striped patterns, such as schematically depicted in Fig. 1, were shown to induce controlled motion of pure liquid droplets over distances up to a few millimeters. Typically, the pattern designs consist of an isotropic hydrophobic region, coated homogeneously with perfluorodecyltrichlorosilane (PFDTS), where the droplet is deposited (top of the linear pattern and centre of the radial pattern in Fig. 1). The gradient is formed by subsequent striped regions (indicated as linear and radial 1, 2 and 3 in Fig. 1) consisting of alternating hydrophobic (PFDTS) and hydrophilic $\left(\mathrm{SiO}_{2}\right)$ stripes with an increasing macroscopic surface energy, which creates a preferential spreading direction for the droplets [38].

The fraction of hydrophilic surface area increases for radial sections further away from the center. We use a dimensionless parameter $\alpha=w / s$ to quantify the relative hydrophobicity of the pattern [41], where $w$ and $s$ represent the hydrophobic PFDTS and hydrophilic $\mathrm{SiO}_{2}$ stripe widths, respectively. Areas with smaller values for $\alpha$ correspond to larger overall surface energy, and as such are more hydrophilic. The range of $\alpha$ values considered in this work amounts to $0.9-0.25$ for all patterns.

In this work, we use such stripe-patterned wetting gradients in an attempt to control the motion of the receding contact line of a droplet containing nanoparticles and with that the deposition of the nanoparticles at the trailing edge of the moving droplet. Moreover, inspired by the recent work of Ahmed and co-workers [42], who observed patterned deposition of gold nanorods on morphologically flat substrates with a linear pattern of hydrophilic stripes, we apply suspensions containing low aspect ratio gold nanorods (and a low concentration of nanospheres) on our gradient substrates. The choice for low aspect ratio nanorods derives from the fact that the shape anisotropy defines a preferential orientation with respect to the drying direction. Moreover, nanorods with an aspect ratio of approximately 6 are relatively easy to synthesize and have been shown to give rise to nicely packed, dense arrays. Considerably larger nanorods will lead to more disordered, randomly piled arrays of the nanorods [42]. After a summary of the experimental procedures, we first review the liquid behavior on the different stripe-patterned surfaces. We discuss the residual liquid film on single stripes as well as the origin of liquid bridges covering several adjacent stripes at regular intervals. In the last section, we describe the deposition of the nanoparticles, which occurs primarily on the hydrophilic stripes. The shape of the deposits on the different patterns is discussed. We also zoom in on individual islands and show that within the micron-scale islands, phase-separation occurs based on size and shape of the different nanoparticle geometries in the suspensions.

\section{Experimental details}

\subsection{Materials}

Cetyltrimethylammonium bromide (CTAB, Aldrich, 98\%), hydrogen tetrachloroaurate $\left(\mathrm{HAuCl}_{4} \cdot 3 \mathrm{H}_{2} \mathrm{O}, 99.999 \%\right.$, Aldrich), silver nitrate $\left(\mathrm{AgNO}_{3}, 99 \%\right.$, Acros), ascorbic acid (AA, 99\%, Merck), sodium borohydrate $\left(\mathrm{NaBH}_{4}, 99 \%\right.$, Aldrich), hydrochloric acid $(\mathrm{HCl}, 37 \%$, Merck) and glycerol (ReagentPlus, Sigma, USA) were all used as received without further purification. All water that was used in the synthesis was of Milli-Q quality $(18.2 \mathrm{M} \mathrm{cm})$, produced in a Simplicity 185 system (Millipore).

\subsection{Substrate preparation}

The surface patterns of self-assembled monolayers (SAMs) of $1 \mathrm{H}, 1 \mathrm{H}, 2 \mathrm{H}, 2 \mathrm{H}$-perfluorodecyltrichlorosilane (PFDTS, 97\%, ABCR, Germany) on silicon wafers are created using standard clean room facilities. First, a positive photoresist is spincoated on freshly cleaned wafers with a natural oxide film, followed by soft-baking. Patterns are created via standard optical lithography, after which the exposed photoresist is washed-off. The remaining photoresist is hard-baked and provides surface protection during vapor deposition of PFDTS; the silane headgroup binds covalently to the native silicon oxide, exposing the fluorinated tail to the liquid. The assembly creates a densely packed layer of molecules with a height in the order of one nanometer, on which both glycerol and water have a stationary contact angle (CA) $\theta_{\text {st }}=106^{\circ}$. Vapor deposition of PFDTS is done in a degassed chamber that is successively exposed to PFDTS and water reservoirs to introduce the respective vapors, initiating the reaction on the wafer surface. After formation of the SAM, the photoresist is washed off, leaving a chemically patterned 


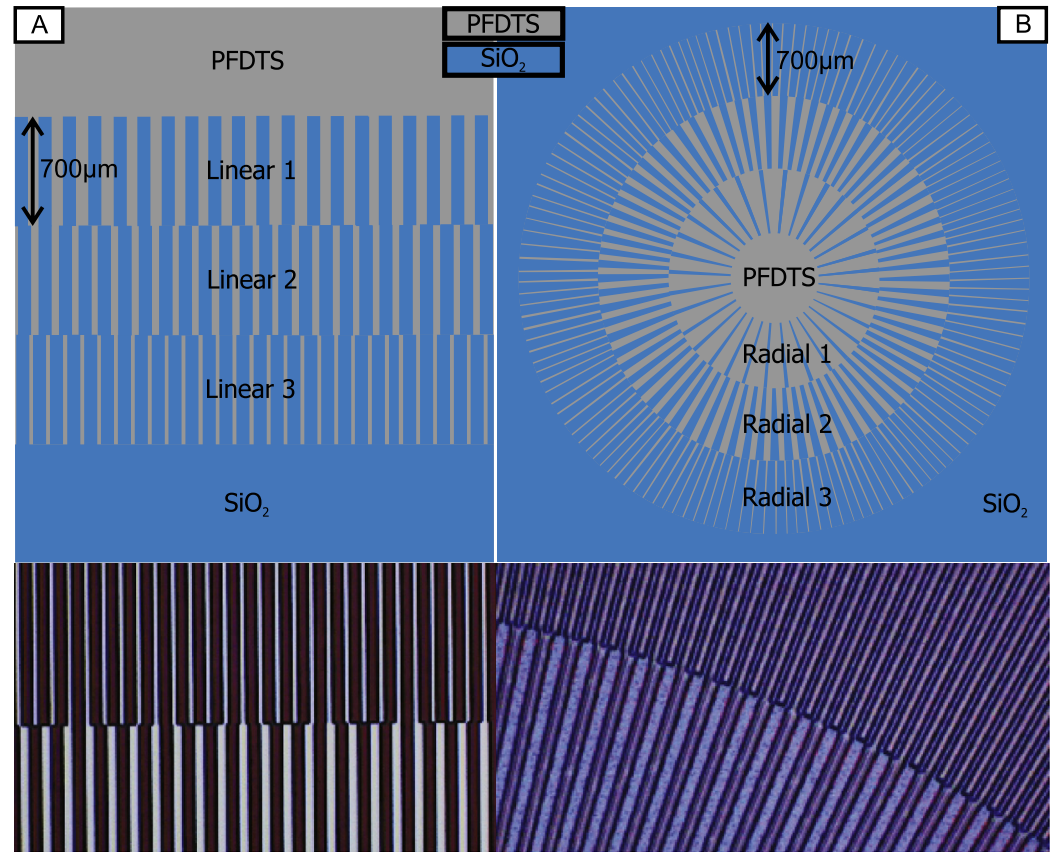

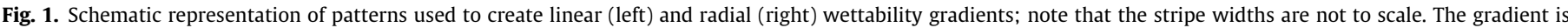

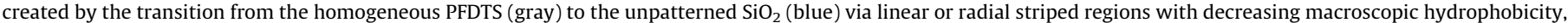

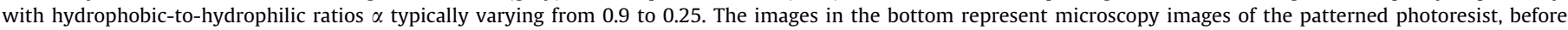

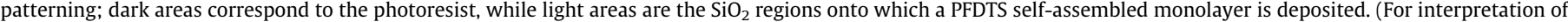
the references to colour in this figure legend, the reader is referred to the web version of this article.)

surface. The uncoated silicon oxide surface exposed after removing the photoresist exhibits typical static contact angles in the range of $30-40^{\circ}$, with receding angles of $10-15^{\circ}$.

\subsection{Nanorod synthesis}

We adopted the two-step seed mediated protocol as described by Nikoobakht and El-Sayed [43] to synthesize the gold nanorods used for this study. First CTAB-coated seed particles were prepared by adding $25 \mu \mathrm{l}$ of $\mathrm{HAuCl}_{4}(0.1 \mathrm{M})$ to $10 \mathrm{ml}$ of CTAB $(0.1 \mathrm{M})$. Next $60 \mu$ l of ice cold $\mathrm{NaBH}_{4}(0.1 \mathrm{M})$ was introduced while continuously stirring for a few minutes. The resulting solution quickly turns light brown, indicating formation of gold seeds. The solution was kept at $25^{\circ} \mathrm{C}$ for approximately one hour without stirring.

As outlined in Section 1, we use gold nanorods with an aspect ratio (AR) of approximately 6 . To synthesize gold nanorods (average length $56 \pm 12 \mathrm{~nm}$ and diameter $10 \pm 1 \mathrm{~nm}$; the size distributions have been determined from measuring 1000 nanorods in SEM images) the growth solution was prepared by adding $50 \mu \mathrm{l}$ of $\mathrm{HAuCl}_{4}(0.1 \mathrm{M})$ to $10 \mathrm{ml}$ of CTAB $(0.1 \mathrm{M})$. At room temperature $20 \mu \mathrm{l}$ of $\mathrm{AgNO}_{3}(0.1 \mathrm{M})$ was added, followed by $70 \mu \mathrm{l}$ of ascorbic acid $(0.1 \mathrm{M})$ with gentle stirring; the resulting solution becomes colorless. Next, $100 \mu \mathrm{l}$ of $\mathrm{HCl}(1 \mathrm{M})$ was added to maintain $\mathrm{pH} \approx 3$. Finally, $24 \mu \mathrm{l}$ of the seed particle suspension was added to the growth solution. This solution was left undisturbed overnight at room temperature.

Before use, the nanorod suspensions were centrifuged at $15,000 \mathrm{rpm}$ for $10 \mathrm{~min}$ to remove the excess CTAB. Subsequently, the suspension was centrifuged again at $5600 \mathrm{rpm}$ for $5 \mathrm{~min}$ to remove spheres from rods. The nanorod suspensions were stored in the refrigerator.

After centrifugation the nanoparticle suspensions contain predominantly nanorods, and a lower concentration of nanospheres. The latter are a residue of the synthesis, but provide interesting effects as to nanoparticle phase-separation [31]. From careful analysis of the relative (transverse and longitudinal) peak heights in UV-vis spectra, we deduce that the nanorod and nanosphere number densities amount to approximately $N_{\text {rods }}=$ $2.0 \times 10^{11} \mathrm{~cm}^{-3}$ and $N_{\text {spheres }}=2.2 \times 10^{10} \mathrm{~cm}^{-3}$, respectively.

\subsection{Liquid deposition and surface characterization}

Droplet deposition is done using an OCA 15+ goniometer (DataPhysics, Germany), employing a computer controlled syringe. Unless otherwise specified, for all droplets the volume is fixed to $1 \mu \mathrm{l}$. The variation in droplet diameter just after being produced from the syringe was measured to be less than $5 \%$. A ccd-camera is mounted within the OCA setup, which enables the capturing of a droplet profile. From this profile the dimensions and the contact angles can be extracted. This is done with the DataPhysics software, resulting in an accuracy of $0.5^{\circ}$ for the contact angles. Deposition of the droplet is achieved by very slowly lowering the syringe with the suspended droplet toward the substrate until it contacts the patterned surface [44]. Droplets of nanorod suspension were placed on the patterned surfaces and allowed to evaporate at room temperature; typically within two hours the solvent was completely evaporated.

A color camera (1.2 MagePixel USB camera, model EO-1312C Edmund Optics, fitted with a close focus zoom lens with a $10 \times$ zoom in combination with $2 \times$ fixed focal length lens extender) is mounted above the deposition stage and is used to image the temporal evolution of the droplets over the patterned surface. The top-view camera has a frame rate of $10 \mathrm{fps}$, enabling a qualitative description of the slow movement of droplets. The color movies provide a means to monitor this residual liquid film.

For morphological characterization, an Asylum Research MFP3D atomic force microscope (AFM) with an $80 \mu \mathrm{m} \times 80 \mu \mathrm{m} \times$ $15 \mu \mathrm{m}$ closed loop scanner has been employed. Measurements were performed under ambient conditions in intermittent contact mode using Olympus AC240TS probes with a cantilever spring 
constant of approximately $2 \mathrm{~N} / \mathrm{m}$ and a resonance frequency of $65 \mathrm{kHz}$. The tetrahedral tips have an opening angle of about $35^{\circ}$ and a tip radius of $7 \mathrm{~nm}$.

High-resolution scanning electron microscopy (SEM; on a Merlin Zeiss 1550 system) was used for imaging of our samples with nanoroparticle deposits; typical voltages in the range $0.1-30 \mathrm{kV}$ are accessible. Images used in this work were obtained at an accelerating voltage of $2 \mathrm{kV}$.

\section{Liquids on chemically stripe-patterned gradients}

\subsection{Experimental results}

We briefly review the gradient-induced droplet motion, as extensively reported in our previous work $[39,40]$. On the linear pattern, the droplet deposited onto the unpatterned PFDTS area initially spreads isotropically. Once the advancing three-phase contact line reaches the first striped pattern, the $\mathrm{SiO}_{2}$ stripes give rise to a higher overall surface energy therewith inducing a preferential spreading direction for the liquid. Sequentially the droplet reaches the following patterns with decreasing $\alpha$ values, i.e. with increasing hydrophilicity (Fig. 1). Droplet motion on radial patterns is also driven by the gradient defined by the ring-shaped striped regions with decreasing $\alpha$ values at larger distance from the center. Generally, the droplets on the radial patterns are markedly more circular as opposed to those on linear wettability gradient arrays. For the linear patterns, the confinement of the liquid between PFDTS stripes is stronger, forcing the liquid to move faster in the stripe direction as compared to the radial patterns considered in this work.

After the droplet has moved over the pattern, the receding side leaves behind a layer of residual liquid. In the case of water, this residual liquid layer evaporated relatively fast, but in the case of glycerol it is stationary. This happens both on the linear and the radial patterns [40]. Close examination of the liquid residue (Fig. 2a) reveals that on the first striped region (radial 1 in Fig. 1), only the hydrophilic $\mathrm{SiO}_{2}$ stripes are covered with the polar liquid. However, on the subsequent striped areas of the pattern, liquid 'bridges' covering multiple stripes (both PFDTS and $\mathrm{SiO}_{2}$ ) can be discerned.

In an attempt to understand the occurrence of bridges on the subsequent striped regions in Fig. $2 b$ and $c$ we zoom in on a part of the radial pattern and the liquid on it. The similar size and regular position of liquid bridges suggests that their presence is probably related to a repeatedly occurring feature in the pattern. As outlined in our previous work [39] the bridges occur where the hydrophilic stripes in the neighboring striped patterns are not connected, i.e. where the periodicity of both patterns is out of registry. Due to the different $\alpha$ values in the two regions the periodicity of the stripes is different, and as such the relative positions of the stripes shift with respect to each other. At positions where bridges are formed, the hydrophobic stripes of the second region originate where hydrophilic stripes of the preceding region end. This perturbes the contact line structure, and as such gives rise to substantial pinning and thus bridge formation. Further enlargement of the border between the two regions (shown in Fig. 2b) reveals a critical mismatch between the respective hydrophobic stripes that will lead to bridge formation.

To assess the actual connection between subsequent striped regions, we performed AFM measurements on the striped patterns. A typical result showing the border between two striped patterns with different $\alpha$ is depicted in Fig. 3. In the composed AFM image, the PFDTS is visible as the 'higher' region (brighter). The top half of the image corresponds to the wider PFDTS stripes, i.e. a more hydrophobic pattern with a higher $\alpha$ values ( $\alpha=0.5$ in this case); the bottom half is more wetting, characterized by $\alpha=0.3$.
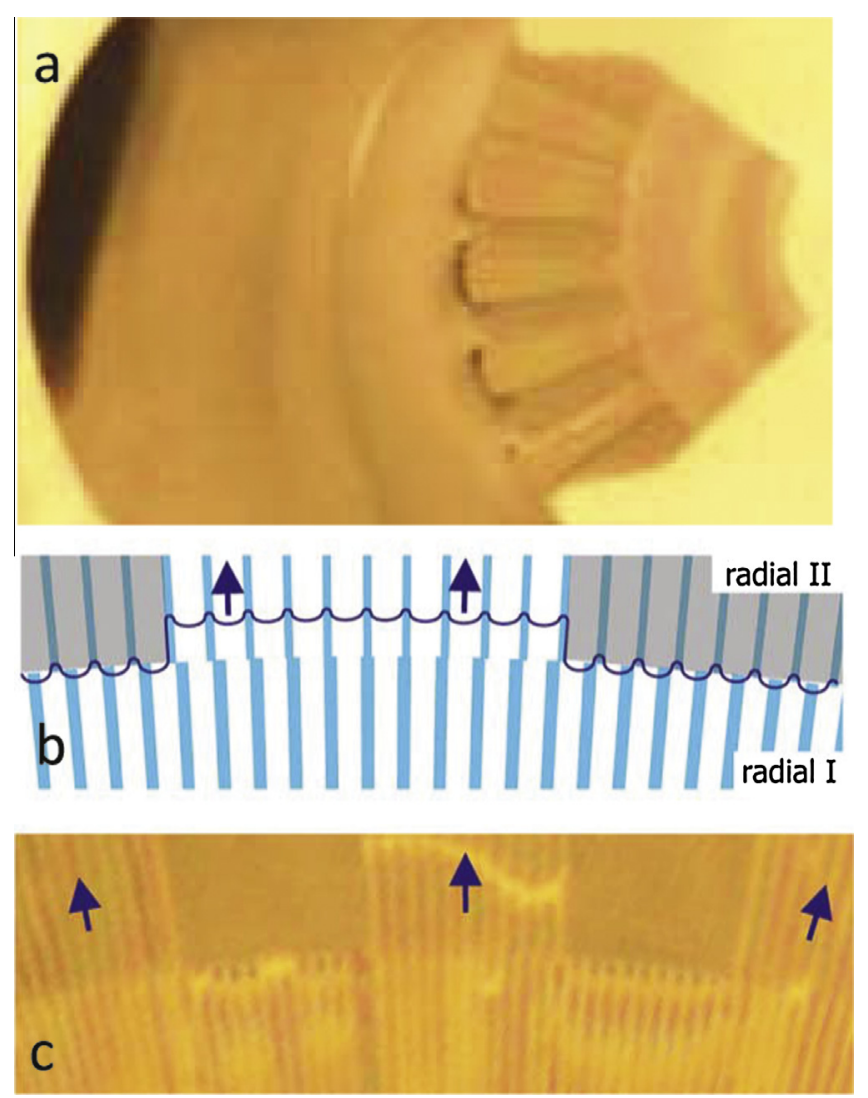

Fig. 2. (a) Image of glycerol remaining on the $\mathrm{SiO}_{2}$ stripes after the droplet has moved outward; the image is obtained several minutes after initial deposition. On the first radial pattern, a homogeneous distribution of glycerol is observed on the hydrophilic stripes. On the second radial pattern, so-called 'bridges' occur, where the liquid covers several stripes. (b) Schematic representation of pinning of the receding contact line at the border between radial patterns (the shaded blue lines correspond to hydrophobic PFDTS, white represents the hydrophilic $\mathrm{SiO}_{2}$ ). The gray rectangles represent the 'bridges', which originate from discontinuities in the PFDTS stripes. (c) Enlarged top-view image of an actual wetted surface, showing the bridges. Image adopted from Ref. [39]. (For interpretation of the references to colour in this figure legend, the reader is referred to the web version of this article.)

\subsection{Modelling liquid 'bridges'}

To verify our assumption that the bridges originate from a mismatch between two neighboring patterns we developed a simple model. In this model the positions of bridges are calculated on the basis of the widths of wetting and non-wetting stripes, as schematically shown in the top panel of Fig. 4. We define the width of the PFDTS and $\mathrm{SiO}_{2}$ stripes on the first and second pattern as $w_{1}, s_{1}, w_{2}$ and $s_{2}$, respectively. As indicated on the basis of our experimental results, to form a bridge the PFDTS stripe of pattern 2 needs to be connected with the $\mathrm{SiO}_{2}$ stripe (in other words, $w_{2}$ needs to be smaller than $s_{1}$ ). If we start with two $\mathrm{SiO}_{2}$ stripes of adjacent patterns aligned (left side in the top panel of Fig. 4) and and we label the number of stripes per pattern with $n_{i}$, where $i$ indicates the number of the pattern, we obtain the following set of equations describing the position of the edges of neighboring stripes

$$
\begin{aligned}
& s_{1 L}=n_{1}\left(s_{1}+w_{1}\right) \\
& s_{1 R}=n_{1}\left(s_{1}+w_{1}\right)+s_{1} \\
& w_{2 L}=n_{2}\left(s_{2}+w_{2}\right)+s_{2} \\
& w_{2 R}=\left(n_{2}+1\right)\left(s_{2}+w_{2}\right)+s_{2}
\end{aligned}
$$

in which $s_{1 L}$ and $s_{1 R}$ denote the positions of the left and right edges of the $\mathrm{SiO}_{2}$ stripes in pattern 1 , and $w_{2 L}$ and $w_{2 R}$ being the positions 


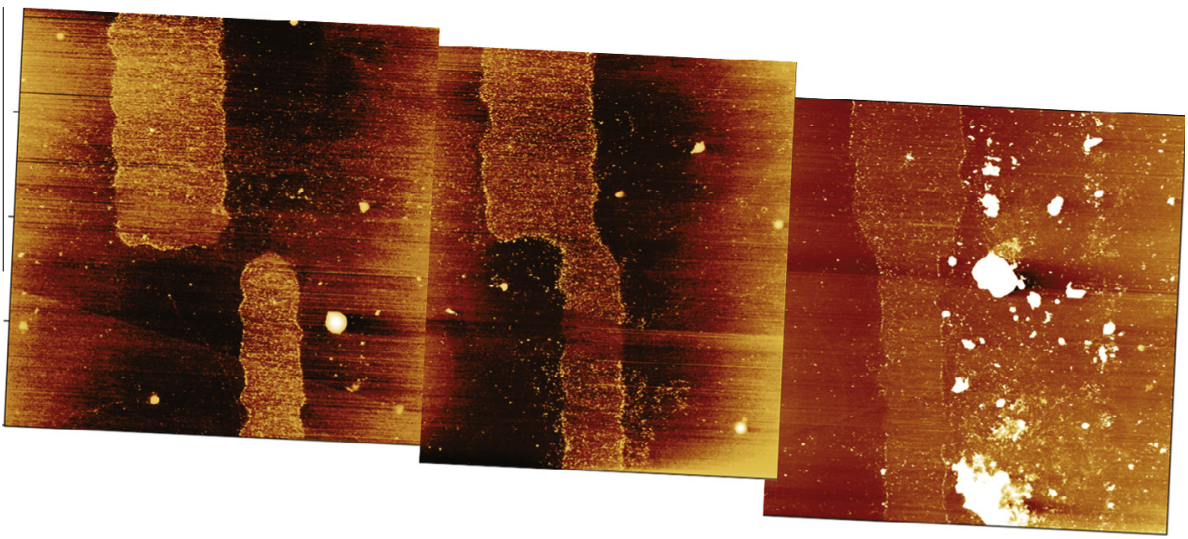

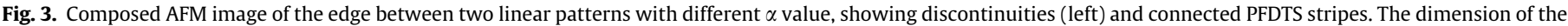
individual square images amounts to $20 \mu \mathrm{m} \times 20 \mu \mathrm{m}$.
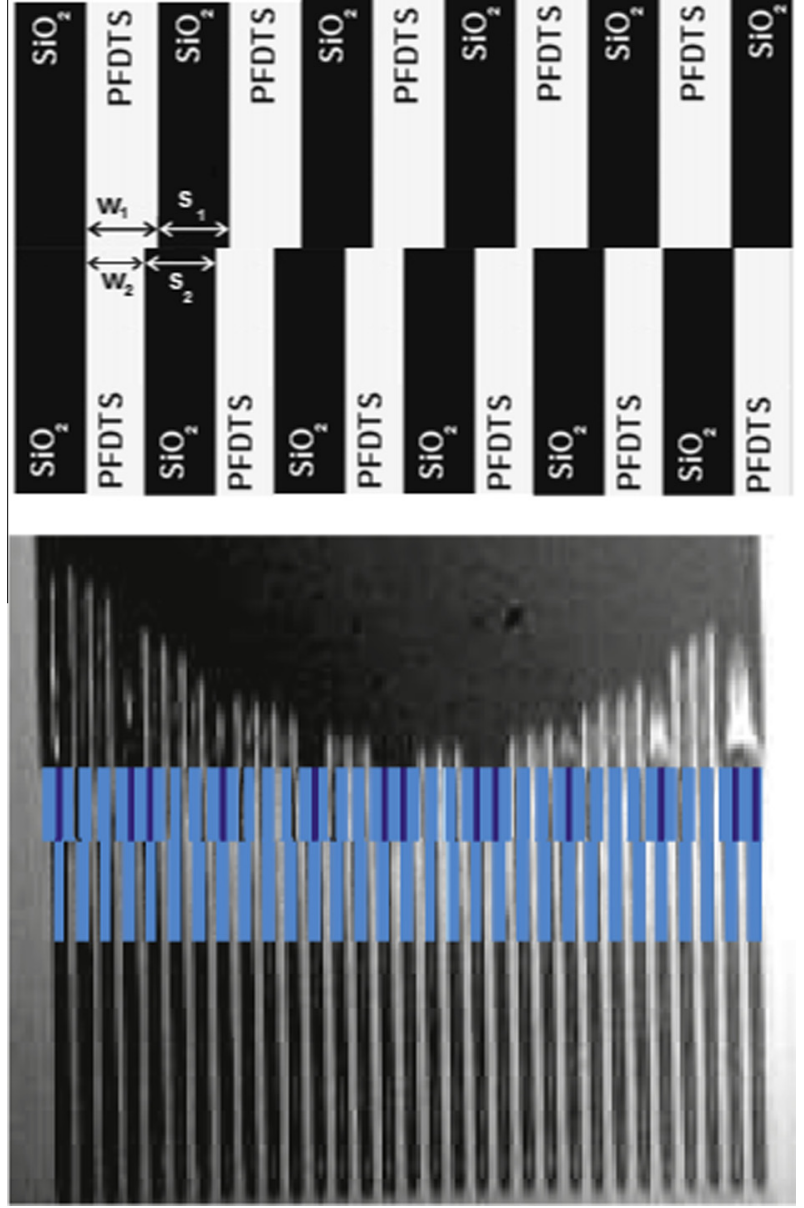

Fig. 4. (top) Schematic overview of the border between two adjacent patterns with dimensions of the PFDTS and $\mathrm{SiO}_{2}$ stripes. (bottom) Snapshot of a droplet on a linear pattern leaving liquid bridges. Overlayed are the $\mathrm{SiO}_{2}$ stripes (light blue) and the calculated positions of the bridges (dark blue); in this case, $s=25 \mu \mathrm{m}, w_{1}=22.5 \mu \mathrm{m}$ and $w_{2}=12.5 \mu \mathrm{m}$. (For interpretation of the references to colour in this figure legend, the reader is referred to the web version of this article.)

of the left and right edges of the PFDTS stripes in pattern 2 . As stated above, in order to form a bridge the position of a PFDTS stripe in pattern 2 needs to be within the position of a $\mathrm{SiO}_{2}$ stripe of pattern 1. Thus

$w_{2 L} \geqslant s_{1 L}$

$w_{2 R} \leqslant s_{1 R}$
After substituting $s_{1}=s_{2}=s$, which is the case for all our patterns, and rewriting, we obtain

$n_{1} \geqslant \frac{n_{2}\left(s+w_{2}\right)+w_{2}}{s+w_{1}}$

$n_{2} \geqslant \frac{n_{1}\left(s+w_{1}\right)-s}{s+w_{2}}$.

Alternatively, we can also use the relative widths of the stripes $\alpha_{i}=w_{i} / s$, i.e. the width $w_{i}$ of the hydrophobic stripes divided by the width $s$ of the hydrophilic stripes:

$n_{1} \geqslant \frac{n_{2}\left(1+\alpha_{2}\right)+\alpha_{2}}{1+\alpha_{1}}$

$n_{2} \geqslant \frac{n_{1}\left(1+\alpha_{1}\right)-1}{1+\alpha_{2}}$.

Rewriting Eq. (4) we obtain an expression for the limits of one integer $\left(n_{2}\right)$ in terms of the other $\left(n_{1}\right)$ :

$\frac{n_{1}\left(1+\alpha_{1}\right)-1}{1+\alpha_{2}} \leqslant n_{2} \leqslant \frac{n_{1}\left(1+\alpha_{1}\right)-\alpha_{2}}{1+\alpha_{2}}$.

For any (positive) integer $n_{1}$ it is straightforward to determine whether there is an integer $n_{2}$, which complies with the limits defined by Eq. (5). If there is such an integer, this will give the position of a bridge.

We implemented this simple model and determined the bridge positions for a specific configuration (Fig. 4), and superimposed it on an actual snapshot in which liquid bridges occur. The calculated liquid residues on the $\mathrm{SiO}_{2}$ are marked in light blue, while the bridges are marked in dark blue (top part of the image). The calculated positions of the bridges align perfectly with the bridges in the snapshot, confirming the validity of our simple model and therewith the origin of the bridges.

\section{Nanorod assembly on stripe-patterned gradients}

\subsection{Nanoparticle deposits}

When a droplet of nanoparticle suspension is placed on a substrate, evaporative assembly generally leads to pinning of the contact line, and the formation of a coffee stain ring [9,28-30]. Using linear or radial wettability gradients, the motion of the droplet and therewith the three-phase contact line can be controlled. Here we primarily focus on the trailing edge of the droplet, and the deposits left by the receding contact line. After the droplet has stopped moving and the solvent has been left to evaporate, typical overview images of the deposits on linear and radial 
stripe-patterned wettability gradients are shown in Figs. 5 and 6, respectively. The gold nanoparticles show up as the brightest features in the SEM images.

Both the linear and radial wettability gradient have a hydrophobic region (consisting of pure PFDTS) on which the droplet is initially deposited (Fig. 1). As soon as the droplet reaches the striped region consisting of alternating hydrophilic/hydrophobic surface, the liquid starts to move toward the other side of the pattern. When the advancing edge reaches the hydrophilic region (pure $\mathrm{SiO}_{2}$ ), the receding edge becomes pinned and overall motion stops.

Comparing the results in Figs. 5 and 6, a number of similarities are observed on both types of patterns. On the advancing side of the droplet, which becomes pinned on the homogeneous $\mathrm{SiO}_{2}$ surface, particle assemblies within the coffee stain ring are observed, very similar to previous work; an example is shown in Fig. 6(C) [31]. Also, on both types of patterns the contact line on the trailing edge of the droplet initially moves a certain distance without leaving behind any deposits; both in the left of Fig. 6(C) and the right of Fig. 6(A), the striped pattern is completely free of deposited nanorods. Close inspection reveals that a few nanoparticles are left behind, but in a significantly lower concentration.

After traveling a certain distance, the receding contact line becomes pinned for the first time. At this position the deposits appear dense and multilayered owing to the pinned contact line and convective transport. The deposits further along the stripes, between the initial multilayered islands and the edge between the two striped regions, the deposits consist mainly of monolayers of nanorods. Furthermore, nanoparticles are only deposited on the hydrophilic stripes; the hydrophilic regions are completely depleted of nanoparticles. Apparently, the deposition is highly selective on the hydrophilic regions of the substrate, most likely due to convective flow during the drying.

At the edge where the different striped areas (with differing $\alpha$ values) meet, dense multilayer deposits are observed on the hydrophilic stripes. As can be seen in Figs. 5(B) and 6(B), the deposits reflect the pinning of the contact line at this edge. Similar to the liquid 'bridges' described in the previous sections, wide deposits covering multiple stripes in the second region occur. Since the brightness is much lower, these are not gold nanoparticles, but most likely $\mathrm{CTAB}$ crystallite layers. Close examination of Figs. 5(B) and 6(B) reveals that these CTAB residues originate on stripes where the hydrophilic stripes in two striped regions do not connect. This supports our conclusions of the previous section. Finally, at the edge between the last striped region and the homogeneous, isotropic hydrophilic region, there is complete wetting. The deposited nanoparticles at the edge and the CTAB on the wetting areas are depicted in Fig. 5(C).

Moreover, on both types of gradient wetting patterns, the nanoparticle assemblies are centered on the hydrophilic stripes. Although it is difficult to follow the exact drying process, we assume that during the final stages hydrodynamic confinement of the liquid gives rise to a force directed toward the middle of the hydrophilic stripes. As a result, the hydrophobic stripes are completely free of nanoparticles, and the nanoparticle islands are centered on the wetting stripes. Moreover, close examination of
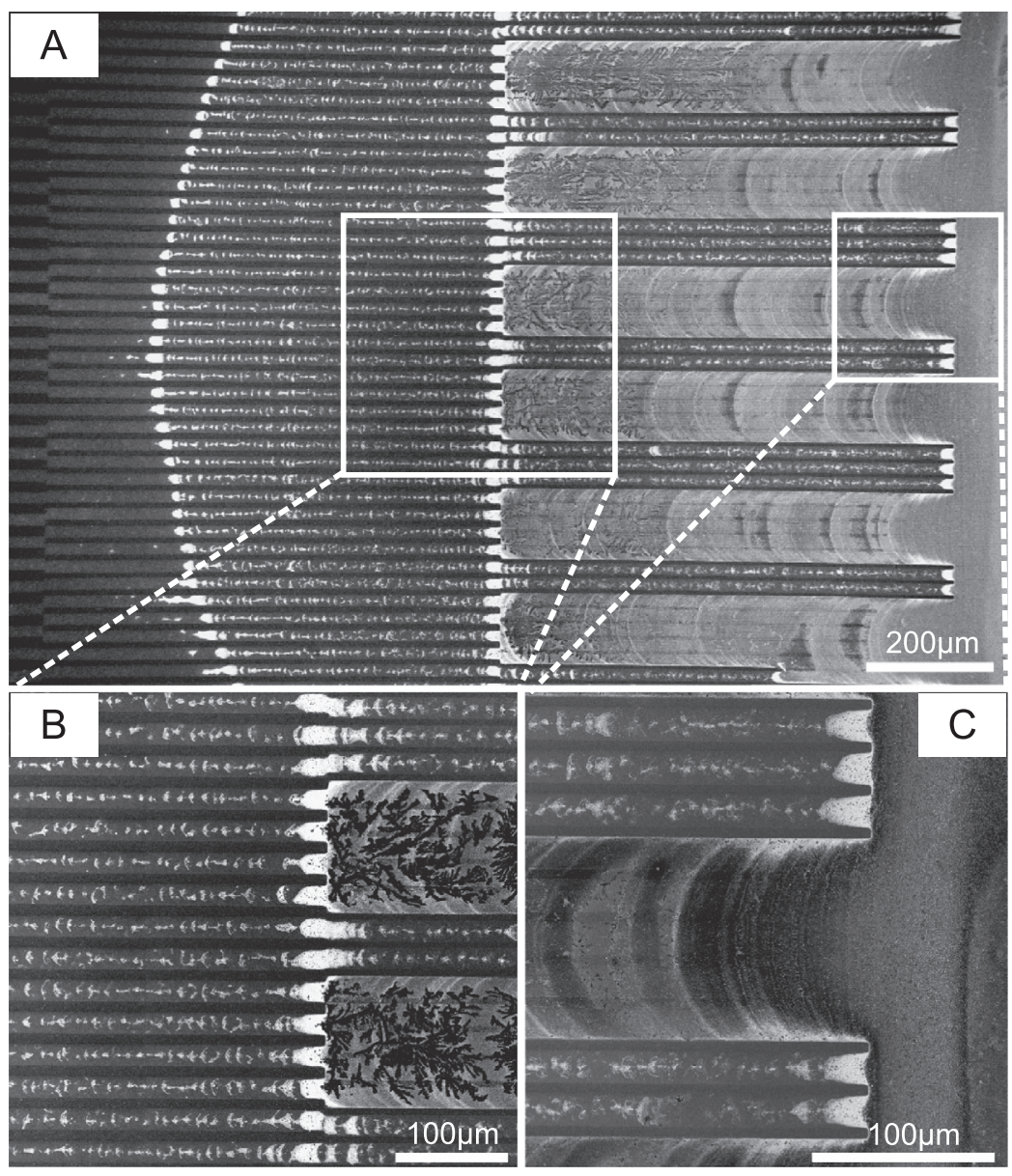

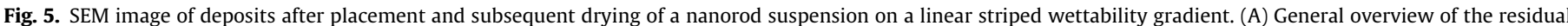

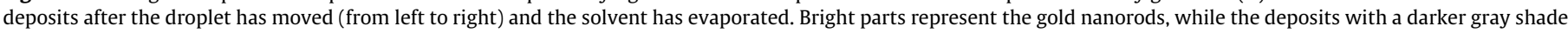
are most likely CTAB layers. (B and C) Zoomed in images of the regions at the border between different striped regions, as indicated in (A). 

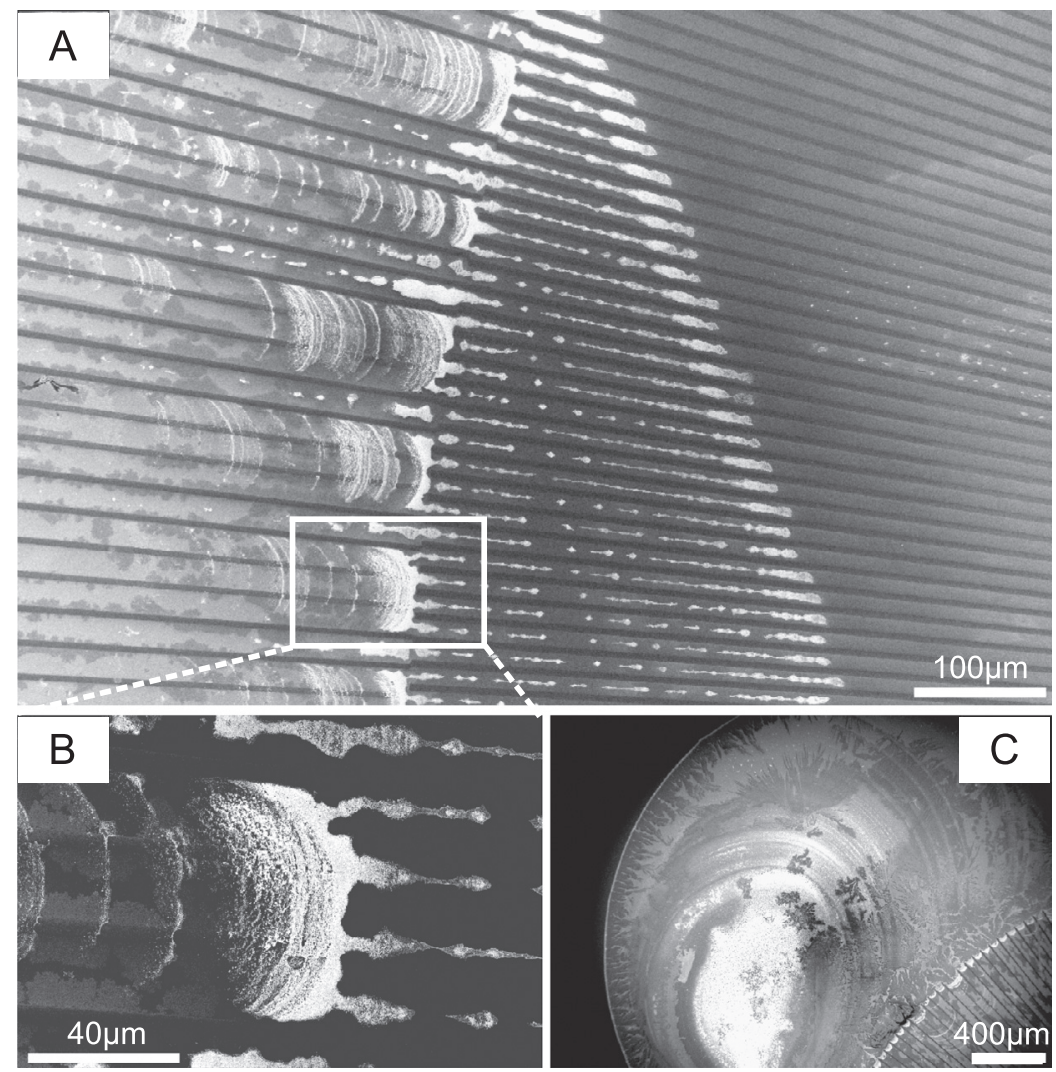

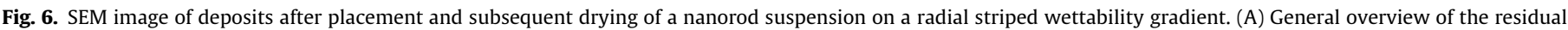

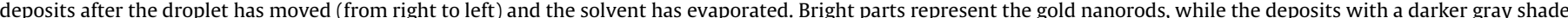

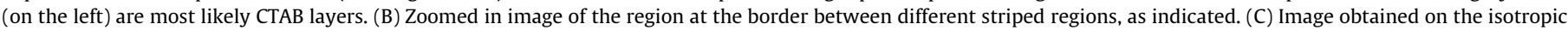
$\mathrm{SiO}_{2}$ region outside the striped radial pattern.

the deposits in Figs. 5 and 6 indeed confirm this assumption in that there is a pronounced symmetry with respect to the middle of the stripes.

There are also differences when comparing the deposited nanoparticle islands on the two striped patterns. On radial patterns the connected deposited islands are relatively longer than the islands on the linear gradient surface. Most of the islands on radial patterned stripes are connected through a thin neck, while on the linear patterns the majority of the islands appear to be isolated, but also on these surfaces we find islands connected through a thin neck.

On linear stripe-patterned gradients the contact line moves in a stick-slip like motion. When the contact line is stuck, or pinned, nanoparticles are assembled at the three-phase contact line. The slippage of the contact line is seen in Fig. 5 by the regions on the hydrophilic stripes between the deposited islands, which have a markedly lower density of nanoparticles. In contrast, on radial patterns the motion of the contact line does not exhibit such pronounced stick-slip like motion. On these surfaces, the deposited islands are often connected by a thin neck.

\subsection{Shape- and size-induced phase separation of nanoparticles}

Finally, in previous work we have shown that the deposition of nanoparticles from mixed suspensions containing nanorods and nanospheres gives rise to phase-separation [31]. Colloidal interactions within the suspension give rise to shape-dependent clustering, followed by convection-driven assembly within the coffee-stain ring. Similarly, the hydrodynamic forces induced by the substrate design also appear to lead to the separation of differently shaped nanoparticles within the deposited islands. In Fig. 7, a typical SEM image of a relatively large island is shown. The trailing part of the island, i.e. on the receding side in the top-right corner of the figure, contains a relatively high portion of nanorods. A monolayer of randomly oriented nanorods has been formed during the drying process. The nanorods within the dashed yellow box have been measured; a histogram of their length distribution is depicted in the top panel of Fig. 7(B). Analysis of the angular distribution reveals there is no preferential orientation.

Moving more toward the center of the deposited island, a ringshaped region containing primarily nanospheres is observed. Within these sphere-rich regions, there appears to be separation of nanospheres with different diameters. To quantify this, a section of the ring has been subdivided into three regions, indicated by the vertical dashed blue lines and the numbers $1-3$. The histograms in Fig. 7(C) indeed confirm that with increasing distance from the edge, the average diameter of the spheres increases from approximately $17 \pm 3 \mathrm{~nm}$ to $27 \pm 5 \mathrm{~nm}$.

Further toward the bottom-left in Fig. 7 a multilayered superstructure containing both spheres and rods is found. Within the dashed red region, the nanorod lengths have been determined. The histogram in the bottom panel of Fig. 7(B) indicates that the nanorods in this part of the droplet are markedly larger than those at the perimeter of the island. Although the phase-separation both in relation to size and shape of the different nanoparticles is obvious from these results, the exact origin remains to be elucidated. We hope that our present findings provide a motivation for further experimental and theoretical work into the evaporation-induced phase separation of nanoparticles. 

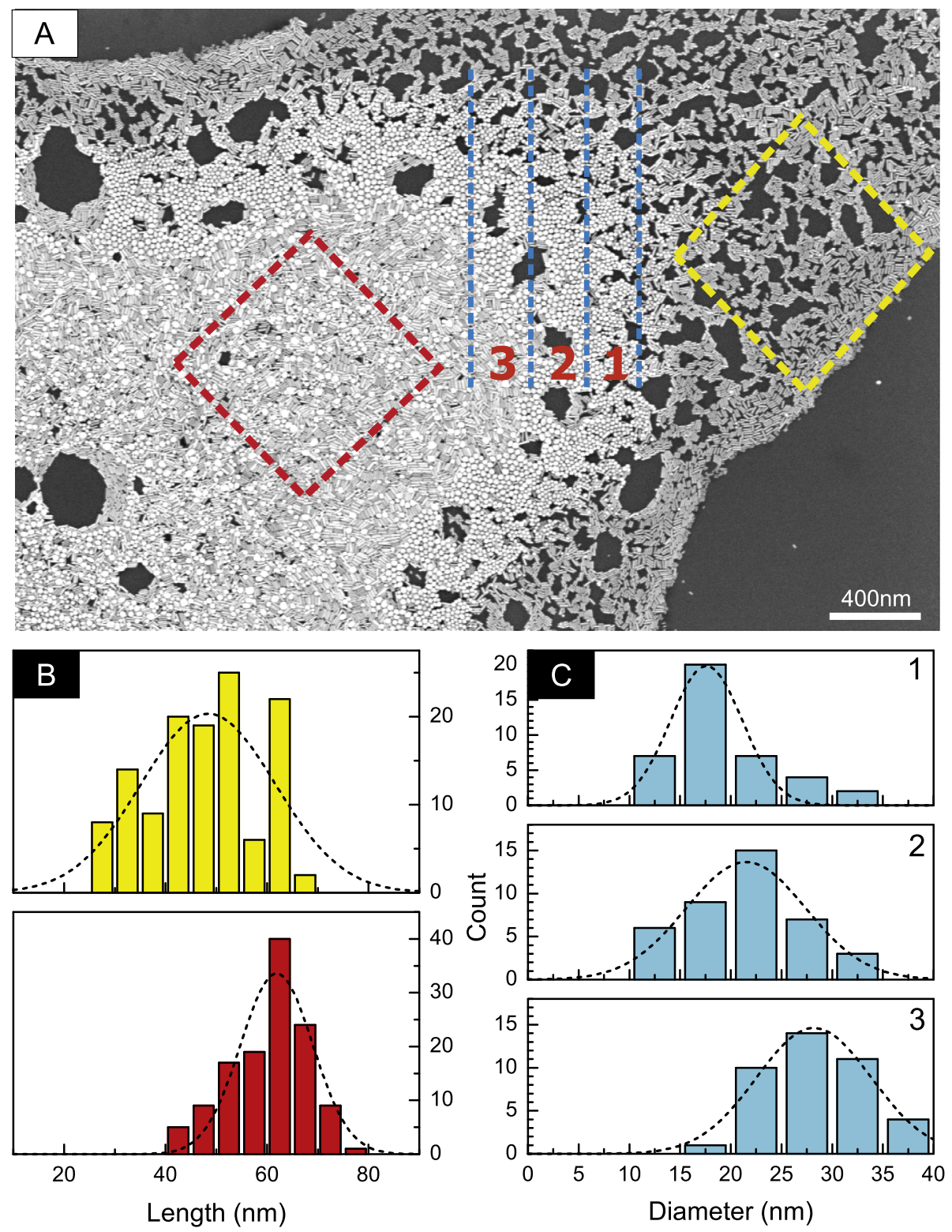

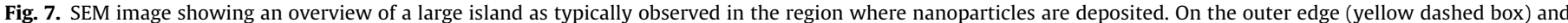

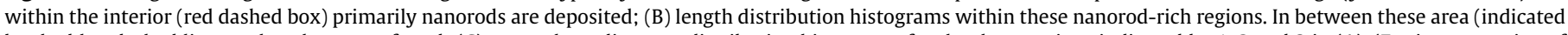

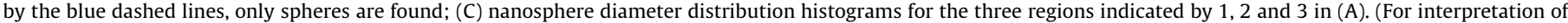
the references to colour in this figure legend, the reader is referred to the web version of this article.)

\section{Conclusions}

We have presented experimental results of work focussed on the use of wettability gradient surfaces to control the motion of liquid droplets and their three-phase contact line. Therewith we explored the potential to use the contact line dynamics as a tool to control nanoparticle deposition over large surface areas. In conventional evaporative self-assembly, nanoparticles generally deposit within the coffee stain ring near the pinned contact line. In this work, we studied the nanoparticle deposits created by the receding contact line at the trailing edge of the moving droplet.

The liquid dynamics on surfaces coated with striped wettability gradients in a linear or radial configuration have been reviewed. Some of the characteristic properties have been reviewed and the occurrence of so-called liquid 'bridges' was evaluated. In previous work it was assumed that these liquid bridges covering multiple stripes originated from pinning of the receding contact line at discontinuities between different striped regions of the pattern. A simple model was developed to test this assumption. Comparison of calculated bridge positions with actual experiments has shown that indeed such a mechanism of pinning on discontinuities is highly probable.

In the second part of the work, we studied the deposits and their spatial distribution left by the receding contact line. SEM images after drying revealed that nanoparticle islands were deposited in the center of the hydrophilic stripes. The remarkably high selectivity gave rise to the hydrophobic areas being almost fully depleted of nanoparticles. A number of similarities between radial and linear stripe-patterned gradients were summarized, and also distinct differences have been addressed. Finally, we also zoomed in on individual islands. Surprisingly a highly selective phase separation was observed within the deposited islands. The outer rim consisted primarily of short nanorods, while more inward a pure nanosphere phase was found. Analysis of the nanosphere 
diameters has shown that more toward the center of the islands larger spheres are assembled. Finally, the interior of the islands consisted of randomly oriented multilayered deposits with nanorods with a substantially larger length as compared to those in the outer rim. A precise mechanism for this effective phase separation is not clear, but we anticipate that our present finding may well pave the way for further work on elucidating its origin.

\section{Acknowledgments}

This work is supported by NanoNextNL, a micro- and nanotechnology consortium of the Government of the Netherlands and 130 partners.

\section{References}

[1] C.B. Murray, C.R. Kagan, M.G. Bawendi, Annu. Rev. Mater. Sci. 30 (2000) 545

[2] A.L. Rogach, D.V. Talapin, E.V. Shevchenko, A. Kornowski, M. Haase, H. Weller, Adv. Funct. Mater. 12 (2002) 653.

[3] F. Caruso, Colloids and Colloid Assemblies: Synthesis, Modification, Organization and Utilization of Colloid Particles, Wiley-VCH, Weinheim, 2004

[4] M.P. Pileni, Nanocrystals Forming Mesoscopic Structures, Wiley-VCH Weinheim, 2005.

[5] X. Ye, L. Qi, Nano Today 6 (2011) 608-631.

[6] V.R. Dugyala, S.V. Daware, M.G. Basavaraj, Soft Matter 9 (2013) 6711-6725.

[7] K. Sefiane, Adv. Colloid Interface Sci. 206 (2014) 372-381.

[8] L.F. Chen, J.R.G. Evans, J. Colloid Interface Sci. 351 (2010) 283-287.

[9] P.J. Yunker, T. Still, M.A. Lohr, A.G. Yodh, Nature 476 (2011) 308-311.

[10] M.A. Hampton, T.A.H. Nguen, Z.P. Xu, L. Huang, V. Rudolph, J. Colloid Interface Sci. 377 (2012) 456-462.

[11] K.M. Carver, R.C. Snyder, Ind. Eng. Chem. Res. 51 (2012) 15720-15728.

[12] F. Meng, M. Doi, Z. Ouyang, Phys. Rev. Lett. 113 (2014) 098301.

[13] Y. Zhou, X. Zhou, D.J. Park, K. Torabi, K.A. Brown, M.R. Jones, C. Zhang, G.C. Schatz, C.A. Mirkin, Nano Lett. 14 (2014) 2157-2161.

[14] S.Y. Zhang, M.D. Regulacio, M.Y. Han, Chem. Soc. Rev. 43 (2014) 2301.

[15] A. Martin, C. Schopf, A. Pescaglini, J.J. Wang, D. Iacopino, Langmuir 30 (2014) $10206-10212$

[16] M.O.A. Erola, A. Partanen, S.C. Okoro, S.M. Rahman, H. Lajunen, S. Suvanto, M Suvanto, M. Kuitinnen, T.T. Pakkanen, Microelectron. Eng. 121 (2014) 76-79.

[17] Z. Hu, M.D. Fischbein, C. Querner, M. Drndic, Nano Lett. 6 (2006) 2585.
[18] C. Ooi, B.B. Yellen, Langmuir 24 (2008) 8514.

[19] T. Klein, A. Laptev, A. Günther, P. Bender, A. Tschöpe, R. Birringer, J. Appl. Phys. 106 (2009) 114301.

[20] W. Ahmed, E.S. Kooij, A. van Silfhout, B. Poelsema, Nano Lett. 9 (2009) $3786-$ 3794.

[21] H.E. Ruda, A. Shik, Nanotechnology 21 (2010) 235502

[22] L.M. Tong, V.D. Miljkovic, M. Kall, Nano Lett. 10 (2010) 268.

[23] C. Hellenthal, W. Ahmed, E.S. Kooij, B. Poelsema, H.J.W. Zandvliet, J. Nanopart. Res. 14 (2010) 1107.

[24] J.W. Liaw, Y.S. Chen, M.K. Kuo, Opt. Exp. 22 (2014) 26005-26015.

[25] L. Zhiguin, Evaporative Self-assembly of Ordered Complex Structures, World Scientific Publishing Co., Singapore, 2012.

[26] S. Semenov, A. Trybala, R.G. Rubiob, N. Kovalchuk, V. Starov, M.G. Velarde, Adv. Colloid Interface Sci. 206 (2014) 382-398.

[27] Z. Zhang, M. Lin, J. Mater. Chem. C 2 (2014) 4545-4551.

[28] C. Nobile, L. Carbone, A. Fiore, R. Cingolani, L. Manna, R. Krahne, J. Phys.; Condens. Matter 21 (2009) 264013.

[29] A.G. Marin, H. Gelderblom, D. Lohse, J.H. Snoeijer, Phys. Rev. Lett. 107 (2011) 085502.

[30] V.R. Dugyala, M.G. Basavaraj, Langmuir 30 (2014) 8680-8686.

[31] I. Ahmad, H.J.W. Zandvliet, E.S. Kooij, Langmuir 30 (2014) 7953-7961.

[32] H.B. Eral, D.M. Augustine, M.H.G. Duits, F. Mugele, Soft Matter 7 (2011) 49544958.

[33] A.W. Wray, D.T. Papageorgiou, R.V. Craster, K. Sefiane, O.K. Matar, Langmuir 30 (2014) 5849-5858.

[34] M.K. Chaudhury, G.M. Whitesides, Science 256 (1992) 1539-1541.

[35] N. Moumen, R.S. Subramanian, J.B. McLaughlin, Langmuir 22 (2006) 2682 2690.

[36] D.J. Huang, T.S. Leu, Appl. Surf. Sci. 280 (2013) 25-32.

[37] C.Y. Lim, Y.C. Lam, J. Phys. D: Appl. Phys. 47 (2014) 425305.

[38] O. Bliznyuk, H.P. Jansen, E.S. Kooij, H.J.W. Zandvliet, B. Poelsema, Langmuir 27 (2011) 11238-11245.

[39] O. Bliznyuk, J.R.T. Seddon, V. Velingura, E.S. Kooij, H.J.W. Zandvliet, B. Poelsema, ACS Appl. Mater. Interfaces 4 (2012) 4141-4148.

[40] E.S. Kooij, H.P. Jansen, O. Bliznyuk, B. Poelsema, H.J.W. Zandvliet, Colloid Surf. A 413 (2012) 328-333.

[41] O. Bliznyuk, E. Vereshchagina, E.S. Kooij, B. Poelsema, Phys. Rev. E 79 (2009) 041601.

[42] W. Ahmed, C. Glass, E.S. Kooij, J.M. van Ruitenbeek, Nanotechnology 25 (2014) $1-10$.

[43] B. Nikoobakht, M.A. El-Sayed, Chem. Mater. 15 (2003) 1957-1962.

[44] O. Bliznyuk, H.P. Jansen, E.S. Kooij, B. Poelsema, Langmuir 26 (2010) 63286334. 